\title{
The organization and temporal properties of appetitive behavior in rats
}

\author{
KATHLEEN M. SILVA and WILLIAM TIMBERLAKE \\ Indiana University, Bloomington, Indiana
}

\begin{abstract}
In the presence and absence of an external interfood clock stimulus (a sequence of flashing lights), rats showed a multimodal behavior pattern during successive quarters of interfood intervals (IFI) ranging from 12 to $192 \mathrm{sec}$. Responses near the feeder peaked before and just after food presentations, whereas locomotion remote from the feeder peaked toward the middle of the IFI. The temporal patterns of nosing in the feeder and remote locomotion were scalar (the time at which a response peaked in the IFI was proportional to the IFI length), whereas the patterns of postfood feeder-directed behavior, rearing, and pawgrooming were time bound (peaking at a fixed time after food, regardless of IFI length). Responses varied in their control by the external clock stimulus. During the last half of the IFI, rats nosed in the feeder more with an external clock, but only at intermediate IFIs. During the first quarter of the IFI, rats pawgroomed more with an external clock, but only at the longest IFI. The general sequence of responses during the interfood clock was consistent with the view that food delivery engages an organized sequence of search states that are expressed through a variety of responses.
\end{abstract}

It is well established that behavior changes predictably during the interval between regular presentations of a reward. For example, during periodic presentations of food, pigeons gradually move away from the feeder after a food presentation and then begin to increase behavior directed toward the feeder wall as the time of the next reward approaches (Staddon \& Simmelhag, 1971; Timberlake \& Lucas, 1985). Rats exposed to periodic access to food generally search near the feeder after eating, then drink, run in a wheel, and return to the feeder near the time of the next food presentation (Lucas, Timberlake, \& Gawley, 1988; Reid, Bacha, \& Moran, 1993).

More precise control of some responses that occur between presentations of food has been achieved by using an interfood clock that consists of a sequence of stimuli that fill the interfood interval (IFI; Dinsmoor, 1994; Dinsmoor, Dougan, Pfister, \& Thiels, 1992; Dinsmoor, Lee, \& Brown, 1986; Ferster \& Skinner, 1957; Matthews \& Lerer, 1987; Palya, 1985; Palya \& Pevey, 1987). For example, Palya (1985) exposed pigeons to a fixed-time (FT) $60-\mathrm{sec}$ schedule in which the IFI was partitioned into ten 6-sec periods, each correlated with a distinctive color on the response key. This procedure reliably initiated keypecking midway through the IFI and accelerated pecking as the delivery of food approached (see, also, Palya \&

This study was facilitated by Grants NSF IBN 9408366 and IBN 9121647 and was part of a dissertation submitted by K.M.S. to the Faculty of Graduate Studies at Indiana University. We thank Jim Dinsmoor, Eliot Hearst, Ellen Ketterson, and Bill Rowland for their helpful suggestions and discussions. Correspondence concerning this article should be addressed to K. Silva, Department of Psychology, University of Redlands, P. O. Box 3080, 1200 East Colton Avenue, Redlands, CA 92373-0999 (e-mail: ksilva@uor.edu).

\footnotetext{
-Accepted by previous editor, Robert A. Rescorla
}

Bevins, 1990). Furthermore, subsequent research demonstrated that pecking begins in the middle of the IFI and reaches a maximum duration just before food, regardless of the IFI duration and the number of clock stimuli, which shows that the onset and peak of keypecking has scalar timing properties --that is, the time at which pecking reaches a maximum increases in proportion to the length of the IFI (Palya \& Pevey, 1987).

Although it is clear that a terminal response, such as pecking, can show scalar properties, the case for nonterminal responses is mixed (Lucas et al., 1988; Palya \& Pevey, 1987). Responses that occur in the middle of the IFI without an interfood clock can show scalar properties (Lucas et al., 1988). For example, Killeen (1975) found that pigeons' general activity peaked approximately onefifth of the way into the IFI, regardless of its length. In contrast, behavior occurring immediately after food may appear time bound to food presentation (Lucas et al., 1988) - that is, the peak of responding occurs at the same absolute time after food, regardless of the length of the IFI (Campagnoni, Lawler, \& Cohen, 1986; Cohen, Looney, Campagnoni, \& Lawler, 1985; Lawler \& Cohen, 1992). Thus, when rats are exposed to different lengths of IFIs, ranging from 16 to $512 \mathrm{sec}$, responses such as waiting by the feeder, rearing, pawgrooming, and drinking reach a maximum level during the same 12-15-sec interval after food presentations and then rapidly decrease (Lawler \& Cohen, 1992; Lucas et al., 1988). However, for a response, such as general locomotion, that predominantly occurs in the middle of the IFI, the time at which it reaches a maximum level increases proportionally with increases in the IFI length.

One framework that predicts the average sequence of responses during the interval between periodic presentations of food is the behavior systems approach (Timber- 
lake, 1983, 1994; Timberlake \& Lucas, 1989; Timberlake \& K. M. Silva, 1995). According to this view, an animal progresses through an organized sequence of search modes (motivational states) between food presentations, in which each mode controls a particular set of responses. For example, immediately after food, a rat is presumed to enter a postfood focal search mode, characterized by rearing, pawgrooming, and sniffing around the feeder area (Lawler \& Cohen, 1992; Lucas et al., 1988). Closer to the middle of the IFI, the rat enters a general search mode that is characterized by locomotion around the experimental chamber. As the time to food delivery approaches, the rat enters a focal search mode that is characterized by behavior directed toward the food site (Lucas et al., 1988). Finally, the animal enters a handling/consuming mode that consists of chewing and ingesting the food. The results of several experiments are consistent with this view of appetitive behavior (Lucas et al., 1988; Reid et al., 1993; Reid \& Staddon, 1990; Timberlake \& Lucas, 1991).

According to a behavior systems approach, if an animal is provided with a predictive stimulus signaling the presentation of food, appropriate search modes should come to be controlled by this stimulus after extended training. The extent to which a stimulus controls a particular search mode should depend on, among other things, the temporal proximity of the stimulus to food. Thus, a stimulus immediately preceding food should come to evoke a focal search mode and appropriate responses, a stimulus presented temporally distant from food should come to evoke a more general search mode and related responses, and a stimulus presented immediately after food should evoke a postfood search mode and related responses (Timberlake, 1994; Timberlake \& Lucas, 1989).

Three issues are not settled in this account. The first is whether the responses associated with the various search modes and clock stimuli will show scalar timing properties. Gibbon and Balsam (1981), in conditioning single conditioned stimuli (CSs), and Palya and Pevey (1987), using interfood clock training, have shown that the initiation of the keypeck response in pigeons is scalar, but little work has been done with other responses and with asymptotic responding. Previous research on adjunctive behavior has provided information about the effect of IFI length on the distribution of multiple responses (Cohen et al., 1985; Lawler \& Cohen, 1992; Lucas et al., 1988), but their relation to external stimuli and the effects of the IFI length on that relation are not clear.

The second issue is whether the presence of external stimuli signaling the temporal proximity to food affects the distribution of behavior, as compared with cases in which the clock is absent. It is reasonable to expect that any temporal pattern might be more pronounced during an interfood clock. The explicit temporal information provided by the clock could be used by the animal to start and stop a search state, as well as for responses. Without the clock, the animal must rely on less distinct temporal cues that may result in a less well defined response distribution. Consistent with this surmise, pigeons show little or no pecking of a key during the interval between noncontingent food presentations without an interfood clock but begin accelerated pecking midway through the IFI when a keylight clock is present (Palya \& Bevins, 1990). However, not all responses during an IFI may be controlled by clock stimuli (Palya, 1993). Matthews, Bordi, and Depollo (1990) have shown with pigeons that responses occurring immediately before and after food presentations are controlled by external cues, but responses occurring in the middle of the IFI are not. Furthermore, it is unknown whether the effects of the external stimuli change as a function of IFI length (see also Lucas et al., 1988; Zeiler, 1991).

The third issue is whether, with training, the elements of an interfood clock can reliably control all elements of the sequence of search modes and responses. Such complex control has several implications for theories of conditioning. In most cases in which the interval between a CS and an unconditioned stimulus (US) is increased beyond a short, optimal length, the probability of the conditioned response decreases (Schneiderman \& Gormezano, 1964). Investigators have viewed this decline in responding as being the result of temporal limitations on excitatory conditioning. Increasing the interval between the CS and the US is assumed to weaken the association between the two stimuli. The behavior systems view argues that it is not due to an absence of control by temporally distant CSs but to their control of a repertoire of responses that differ from those typically measured.

The purpose of the present study was to (1) determine the temporal pattern of multiple responses of rats during an IFI, (2) examine the effect of the presence as opposed to the absence of the interfood clock on these responses, and (3) determine which responses during the interfood clock showed scalar timing properties (responding proportional to the length of the IFI) as opposed to timebound properties (responding at an absolute elapsed time). To address these issues, five groups of rats were exposed to an interfood clock (Groups C12, C24, C48, C96, and C192). The interfood clock consisted of a sequence of four distinguishable visual stimuli (lights that flashed at different rates), the last of which was followed by food (i.e., S1-S2-S3-S4 food). These clock groups differed in the length of the IFIs and the length of the clock stimuli. Five other groups (Groups NC12, NC24, NC48, NC96, and $\mathrm{NC192}$ ) were exposed to IFIs of the same length as the clock groups, but without the interfood clock.

The six responses measured were (1) nosing in the feeder, (2) stepping on a platform in front of the feeder, (3) stepping on a platform remote from the feeder, (4) rearing, (5) pawgrooming, and (6) contacting a lever located beside the feeder. These responses were chosen on the basis of previous research that exposed rats to periodic food presentations (see, e.g., Lawler \& Cohen, 1992; Lucas et al., 1988, Reid et al., 1993), of observations of rats in natural environments (see, e.g., Barnet, 1975), 
and of frameworks that predict the form of responses during different portions of the IFI (Cohen et al., 1985; Timberlake \& Lucas, 1989; Timberlake \& K. M. Silva, 1995 ). We also attempted to measure behavior directed toward the clock stimuli by locating a lever underneath the light source of the interfood clock. Previous research showed that, if rats approach a light, they often contact such a lever with their paws (F. J. Silva, Timberlake, \& Koehler, 1996).

On the basis of the behavior systems framework and of previous research that examined the effect of IFI length on the pattern of responding during interfood intervals without clocks (Cohen et al., 1985; Lawler \& Cohen, 1992; Lucas et al., 1988), we expected that, on average, different responses should peak during different portions of the interfood clock. Postfood behavior, such as standing on the platform in front of the feeder (without putting the head in the feeder), rearing, and pawgrooming, should reach a maximum just after food presentations. Locomotion on a platform remote from the feeder (general search behavior) should peak during the middle clock stimuli, and nosing in the feeder (focal search behavior) should peak just before food presentations. The patterns of responses during all interfood clocks were compared in order to obtain information about the time-bound and scalar characteristics of different responses. If a response has scalar properties, the time at which it reaches its average maximum level (i.e., peaks) should increase in proportion to the increase in IFI length. For example, if a scalar response peaks during the middle portion of the 12-sec IFI, it should also peak during the middle portion of longer IFIs. However, if a response is time bound, the time at which it peaks should be the same, regardless of IFI length.

\section{METHOD}

\section{Animals}

Eighty naive female Sprague-Dawley albino rats, approximately 90 days old at the start of the experiment, were maintained at $85 \%$ of their free-feeding weights throughout the experiment. Outside an experimental session, the rats were housed in individual home cages in a colony room regulated by a 12:12-h light:dark cycle. They were fed approximately $12 \mathrm{~g}$ of commercial rat chow in their home cages after each experimental session in order to maintain them at $85 \%$ of their free-feeding weight. Water was always available in their home cages.

\section{Apparatus}

A schematic of the apparatus is shown in Figure 1. The rats were tested in four identical chambers. Each chamber was a rectangular sheet metal box $60 \times 30 \times 30 \mathrm{~cm}$ with a Plexiglas top and front. The food receptacle was $2.0 \times 5.0 \times 1.8 \mathrm{~cm}$ deep and located on a side wall $12 \mathrm{~cm}$ toward the back of the chamber. Food reward was delivered by a Waltke feeder (Waltke Scientific Enterprises, Psychology Department, Indiana University), which delivered two 45mg Noyes pellets successively into the feeder. An infrared beam projected across the entrance of the feeder; an $11.0 \times 10.0 \mathrm{~cm}$ platform was located on the floor in front of the feeder; and a $9.0 \times$ $14.0 \mathrm{~cm}$ platform was located on the floor along the back of the chamber. A retractable lever was located on the feeder wall, $5 \mathrm{~cm}$ from the back of the chamber and $4 \mathrm{~cm}$ above the floor. The clock stimuli were presented by a green $28-\mathrm{V}$ jewel light $2 \mathrm{~cm}$ above the lever.

The experimental room also contained a video camera that monitored the experimental chambers. The video camera was connected to a VCR that recorded experimental sessions and to a monitor for closed-circuit observation. Data-logging, control equipment, and the VCR were located in an adjacent room. The experimental chambers and the programming and recording equipment were located in separate rooms.

\section{General Experimental Procedures}

Experimental sessions were conducted at the same time daily, 6 days per week. There were 20 IFIs. Each session terminated immediately after the 20th IFI. The experiment consisted of three phases: chamber adaptation, feeder training, and food delivery after fixed intervals of time.

\section{Chamber Adaptation}

The rats were put in the chambers for 10 min on 2 successive days. No food or stimuli were presented. In their home cages, the rats were fed the same number of food pellets that they received during later phases.

\section{Feeder Training}

Five pellets were placed on the platform in front of the feeder at the beginning of a session, and 20 pellets were placed in the feeder. A session terminated after the rats had eaten all of the pellets or

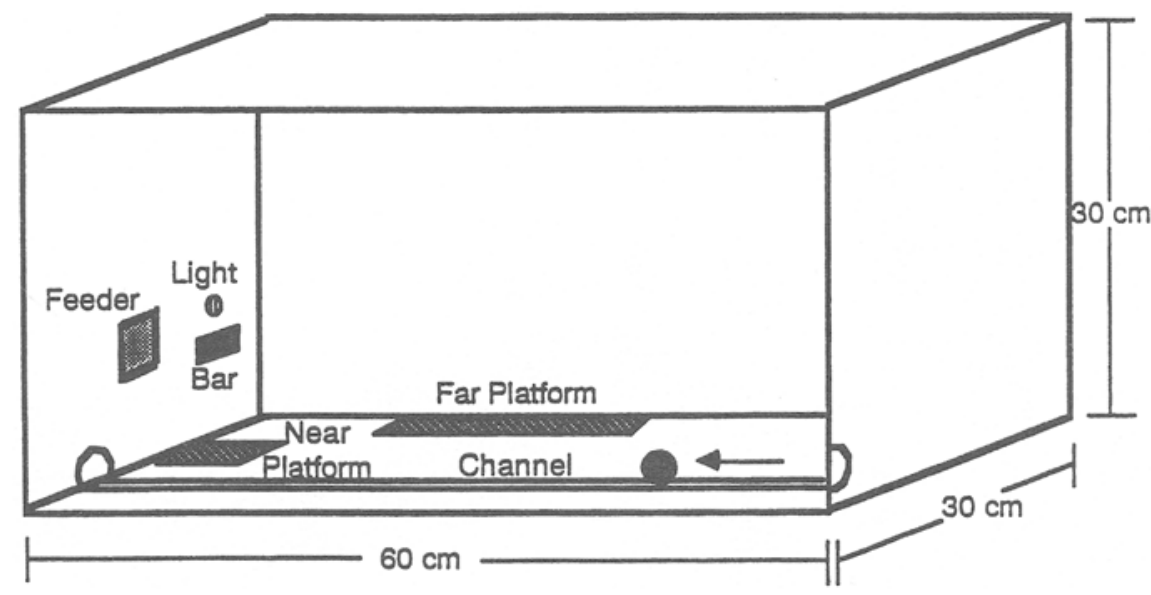

Figure 1. A schematic of the apparatus. 
after $30 \mathrm{~min}$ had elapsed, whichever occurred first. A rat was considered trained when it ate 20 food pellets within a 30 -min session for 2 consecutive days.

\section{Interfood Clock Groups}

Rats in Groups C12, C24, C48, C96, and C192 were exposed to 16 sessions with an interfood clock that consisted of a sequence of four 3-sec lights (Group C12), 6-sec lights (Group C24), 12-sec lights (Group C48), 24-sec lights (Group C96), or 48-sec lights (Group C192) flashing at different rates. The first light (S1) did not flash; the second light (S2) flashed on:off at a rate of $1 \mathrm{sec}$; the third light (S3) flashed on:off at a rate of $0.5 \mathrm{sec}$; the fourth light (S4) flashed on:off at a rate of $0.1 \mathrm{sec}$. Two food pellets were presented immediately after the offset of the last stimulus (i.e., S1-S2-S3S4-food). The order of the flashing lights was not counterbalanced in the present experiment. However, subsequent experiments that counterbalanced for order of the lights showed that the same pattern of responses occurs, whether the stimuli flash progressively faster, flash progressively slower, or occur in a mixed order (K. M. Silva, 1996). In order to stimulate additional attention toward the clock stimuli, the lever was moved into the chamber at the onset of a clock stimulus and out of the chamber at the offset of a clock stimulus.

\section{No-Clock Groups}

The rats in Groups NC12, NC24, NC48, NC96, and NC192 were exposed to 16 sessions, in which the IFIs were $12,24,48,96$, and $192 \mathrm{sec}$, respectively. Clock stimuli were not presented to these groups. The lever extended into the chamber at the beginning of the session and remained there until the end of the session in order to minimize any external timing cues that might predict the delivery of food. Subsequent experiments have shown that the temporal pattern and absolute levels of lever contact for no-clock groups receiving lever presentations in this manner did not differ from those of no-clock groups in which the lever was retracted and reinserted in the chamber every quarter of the IFI (as was the case for the clock groups).

\section{Dependent Measures and Data Analysis}

Four dependent measures were automatically obtained by computercontrolled devices: the durations of nosing in the feeder, activating a platform located relatively far from the feeder (i.e., the far platform), activating a platform located relatively near to the feeder (i.e., the near platform), and contacting the lever located beside the feeder. Two other dependent measures, rearing and pawgrooming, were coded from videotape of the last session. These responses typically occurred shortly after food presentations and provided additional information about a rat's behavior during the IFI that could not be ascertained from the automated measures. Rearing was operationally defined as standing on the hind legs, with or without touching the chamber wall and without grooming. Pawgrooming was defined as licking or biting the front paws. Although the Results section shows responding only for the end of training, the subjects reached stable levels of responding for all automated dependent measures by the 10th day of acquisition. Observation of experimental sessions revealed that the topographical measures (rearing and pawgrooming) were also stable by the 10th day of acquisition.

The Results section includes the data from responses that were automatically measured (nosing in the feeder and activity on the far and near platforms), averaged over the last 4 days of training. Because of the extensive amount of coding involved in measuring rearing and pawgrooming, the data from these responses are averaged over all trials of the last day of training. For these two responses, interobserver agreement (IOA) was calculated on $35 \%$ of the trials for each group-a value that is consistent with recommendations made by Kazdin (1982). An agreement of $90 \%$ was reached on these trials.

Because it was very rare that the rats nosed in the feeder without standing on the near platform, the time spent standing on the near platform initially reflected the total time the rats spent standing on the platform while nosing in the feeder and while not nosing in the feeder. To separate standing-on-the-platform nosing in the feeder from standing-on-the-platform not nosing in the feeder, the amount of time spent nosing in the feeder was subtracted from the amount of time spent standing on the near platform. Thus, the amount of time that the rats spent on the near platform excludes the amount of time that the rats were on the platform while nosing in the feeder. The type of statistical analyses that were used are described in the Results section. All significant effects are reported at the $p<.05$ level.

\section{RESULTS}

\section{Presence Versus Absence of the Interfood Clock}

A major purpose of the present experiment was to determine the effect of the presence of the clock stimuli on responding, as compared with a condition in which the clock was absent. In general, the clock stimuli could affect two response characteristics: its absolute level and its pattern across the IFI. To show how the clock affected these aspects of a response, each panel in Figures 2-6 shows the percentage of time that groups of rats exposed to a clock (clock groups) and not exposed to a clock (noclock groups) spent nosing in the feeder, on the near platform, pawgrooming, on the far platform, and rearing. In each figure, the patterns of responding across the IFI represent responding averaged across all 8 rats in a group. Although not shown, the data of individual subjects were typically similar to those of the average of the group. Exceptions to this are described below. Since lever contact was very low or absent and remained unchanged across all IFIs, with and without clock stimuli, these data are not shown and were excluded from subsequent analyses.

Three-way analyses of variance (ANOVAs) examined the influence of IFI length $(12,24,48,96$, and $192 \mathrm{sec})$, group type (clock, no-clock), and quarter of the IFI (i.e., a particular clock stimulus for clock groups and the comparable IFI time period for the no-clock groups) in order to determine whether there were any three-way interactions for any of the dependent measures. The ANOVAs conducted on nosing in the feeder, pawgrooming, and activity on the near platform showed such interactions $[F \mathrm{~s}(12,210)>2.39]$. Because significant three-way interactions were found, individual two-way ANOVAs that examined the influence of group type (clock vs. no-clock) and segment of time in the IFI (i.e., clock stimulus for clock groups vs. the comparable time period for the noclock groups) were conducted on data from each IFI length in order to determine at which lengths the differences occurred. To clarify the relevant comparisons of the twoway ANOVAs, a detailed description of the analysis is provided for the first dependent measure-nosing in the feeder. The same types of analyses were conducted on the pawgrooming and near platform activity data; however, detailed description of the analyses is omitted in order to facilitate reading of the results.

Figure 2 shows that nosing in the feeder increased during the last half of the IFI for all clock and no-clock groups; however, Groups C24 and C48 showed sharper 


\section{NOSING IN FEEDER}
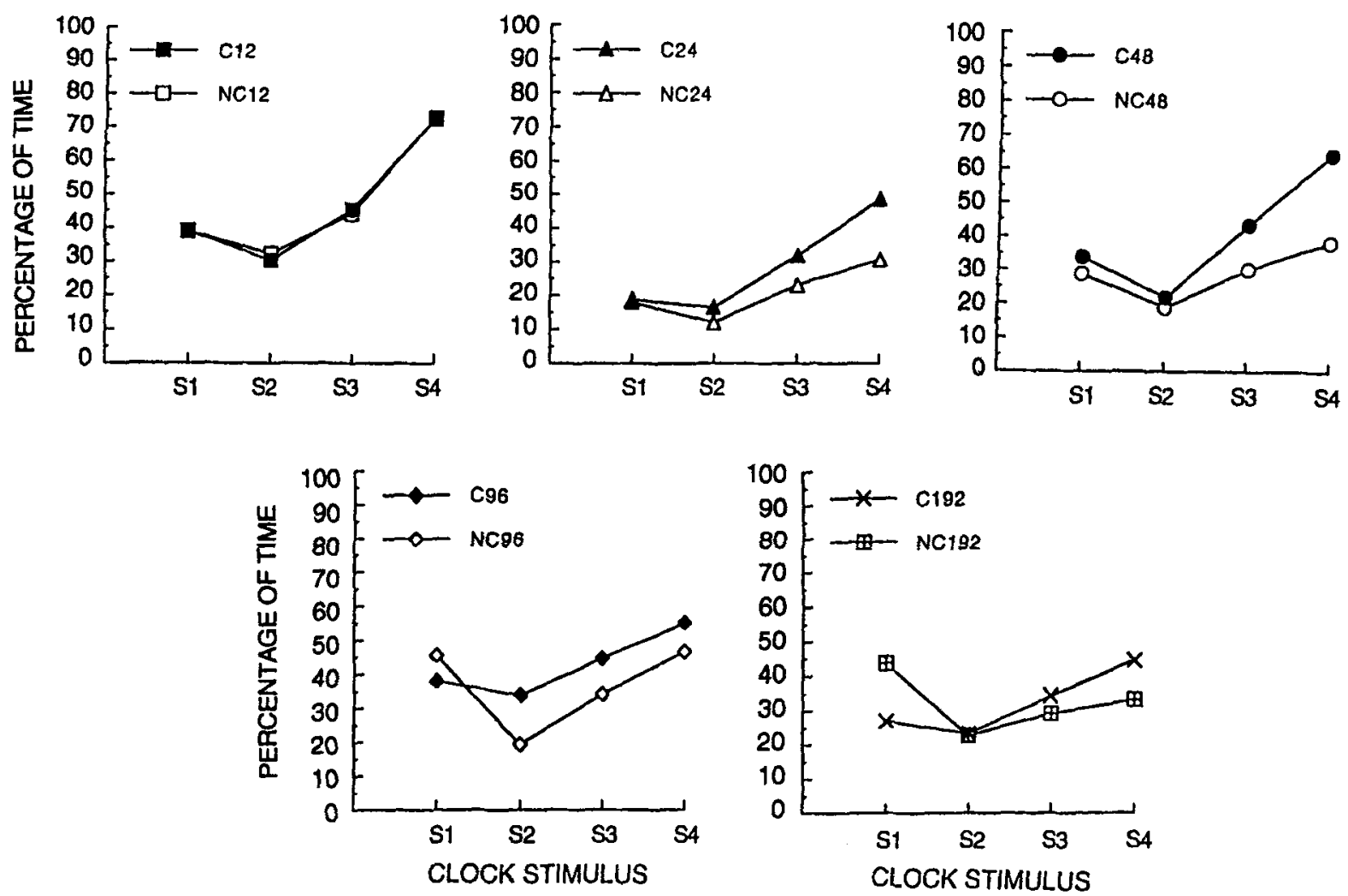

Figure 2. Mean percentage of time spent nosing in the feeder for each clock group and its corresponding no-clock group, averaged across the last 4 days of interfood clock training.

increases than did their no-clock counterparts. Furthermore, Groups $\mathrm{C} 24$ and $\mathrm{C} 48$ nosed in the feeder more than did their respective no-clock counterparts during the last half of the IFI. The statistical analyses described below confirmed these results. A significant main effect of segment of the IFI during all IFI lengths $\left[F_{\mathrm{s}}(3,42)>52.69\right]$ showed that nosing in the feeder changed across the IFI for each clock group and for its no-clock counterpart. Individual Scheffé tests revealed that all of the clock groups and their no-clock counterparts exposed to the 12-, 96-, and 192-sec IFIs increased nosing in the feeder between S2 and S4. Although Groups NC24 and NC48 also showed this increase, Groups C24 and C48 showed an increase from $\mathrm{S} 2$ to $\mathrm{S} 3$ as well as from $\mathrm{S} 3$ to $\mathrm{S} 4$. Thus, during the 24- and 48-sec IFIs, the presence of the clock resulted in a greater increase in responding across the last half of the IFIs, as compared with cases in which it was absent.

A significant interaction between group type and segment of IFI for the 24- and 48-sec IFIs $[F \mathrm{~s}(3,42)>5.74]$ suggested that a clock stimulus and its no-clock counterpart differed during some segments of these IFI lengths. To identify during which clock stimuli the differences occurred, one-tailed independent samples $t$ tests were conducted for each clock stimulus. The results revealed that Groups $\mathrm{C} 24$ and $\mathrm{C} 48$ spent more time nosing in the feeder during the last two segments of the IFI than did their noclock counterparts [ts $(14)>1.77]$. Although Groups C96 versus $\mathrm{NC} 96$ and $\mathrm{C} 192$ versus $\mathrm{NC} 192$ showed a similar trend, the differences between the respective clock and no-clock groups were not statistically significant. In fact, 2 rats from Group C96 and 1 rat from Group C192 responded at a relatively high level, which inflated the group averages. In sum, these data suggest that nosing in the feeder begins to increase in the middle of the IFI and peaks just before food presentations, whether the clock is present or not. However, at intermediate IFIs of 24 and $48 \mathrm{sec}$, the presence of the clock facilitates responding during the last half of the IFI and produces a sharper increase in responding across the IFI, as compared with cases in which it is absent.

Pawgrooming (shown in Figure 3) peaked soon after food presentations for each clock and no-clock group and decreased as the IFI elapsed $\left[F_{\mathrm{s}}(3,42)>4.14\right]$, except for Groups $\mathrm{C} 12$ and $\mathrm{NC} 12$, which showed little change in this response across the IFI. The clock groups and their no-clock counterparts exposed to the 24-, 48-, and 96-sec IFIs all showed similar decreases in responding between the second and fourth segments of the IFI, which suggests that the presence of the clock had little effect. Scheffé tests confirmed that pawgrooming decreased 


\section{PAWGROOMING}
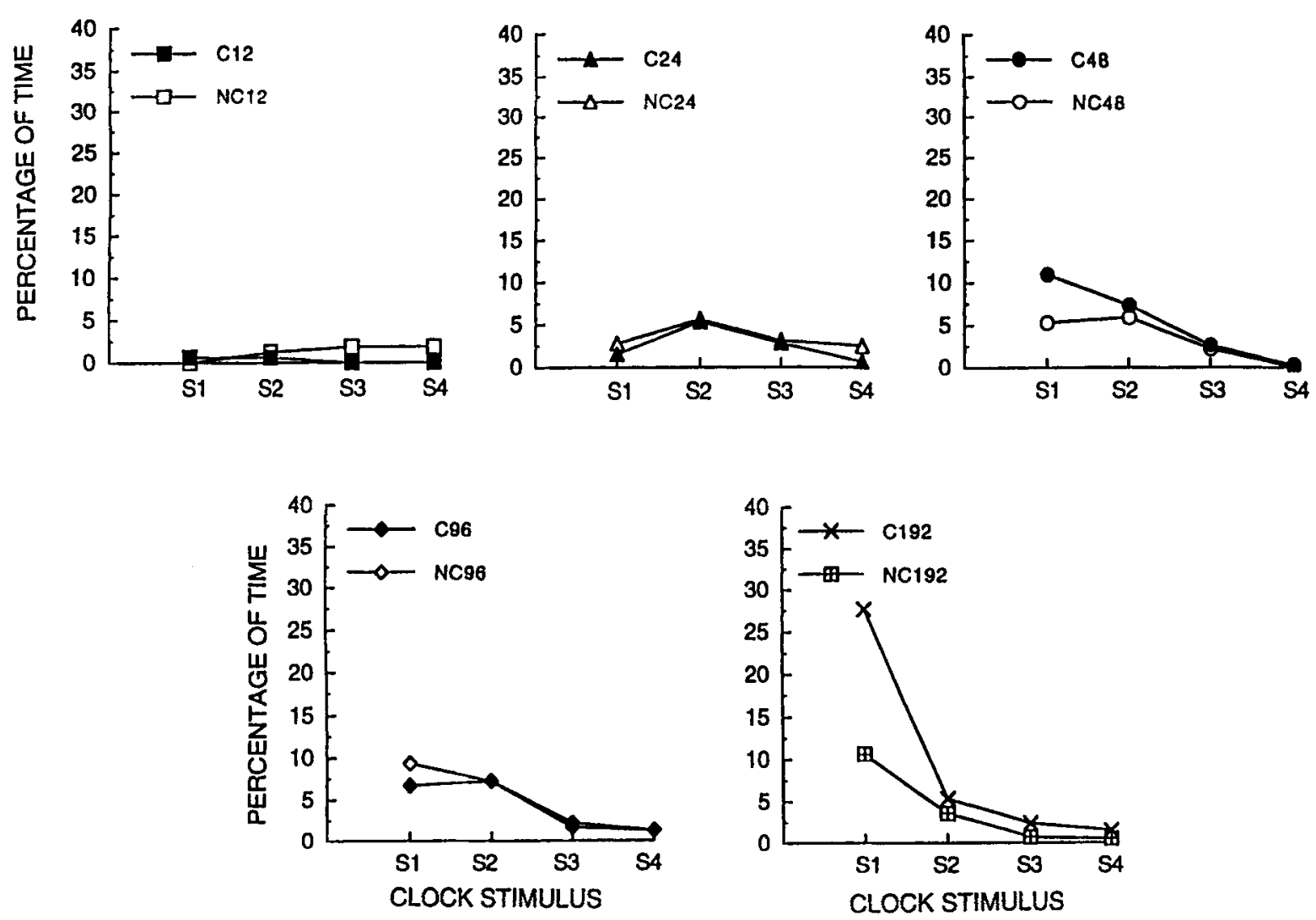

Figure 3. Mean percentage of time spent pawgrooming for each clock group and its corresponding no-clock group during the last day of interfood clock training.

between the second and fourth segments for each clock and no-clock group during these IFI lengths. An interaction between group type and time in the IFI was found only during the 192-sec IFI $\left[F_{\mathrm{S}}(3,42)>3.19\right]$. Scheffé tests revealed that Group NC192 showed a decrease in pawgrooming between S1 to S4, whereas Group C192 showed a decrease between S1 and S2. Independent samples $t$ tests conducted during each clock stimulus revealed that Group C192 spent more time pawgrooming during the first segment of the IFI than did its no-clock counterpart $[t(14)=2.93]$. Together, these data show that pawgrooming peaks after food presentations and gradually decreases across the IFI, whether the clock is present or not. However, at the longest IFI length of $192 \mathrm{sec}$, the presence of the clock facilitated responding and produced a sharper decline in responding across the IFI.

With respect to activity on the near platform (shown in Figure 4), Groups $\mathrm{C} 12$ and NC12 decreased activity across the IFI, whether the clock was present or not $[F(1,14)=$ 11.37]; however, for the other groups, activity on the near platform changed differently across the IFI for clock and no-clock groups. That is, there was an interaction between group type and time in the IFI for the other IFI lengths $[F \mathrm{~s}(3,42)>3.90]$. Scheffé tests conducted on the data from each clock group and its no-clock counterpart revealed that all of the clock groups decreased activity on the near platform across the IFI, whereas the platform activity for the no-clock groups did not change.

In terms of the absolute levels of responding during individual clock stimuli, Groups NC24, NC48, NC96, and NC192 spent more time on the platform in front of the feeder during the last half of the IFI than did their clock counterparts. Independent samples $t$ tests revealed that Groups NC24 and NC48 spent more time on the near platform during the last segment of the IFI than did their clock counterparts, and Groups NC96 and NC192 spent more time on the platform during the last two segments of the IFI than did their clock counterparts $[t \mathrm{~s}(14)>2.10]$. Together, these data suggest that the clock had little effect on activity near the feeder during the shortest IFI. However, during the remaining IFI lengths, the presence of the clock suppressed this response (in favor of nosing in the feeder) during the last half of the IFI.

Although clock stimuli facilitated nosing in the feeder and pawgrooming and suppressed activity on the near platform, they had little effect on the two remaining mea- 


\section{NEAR PLATFORM}
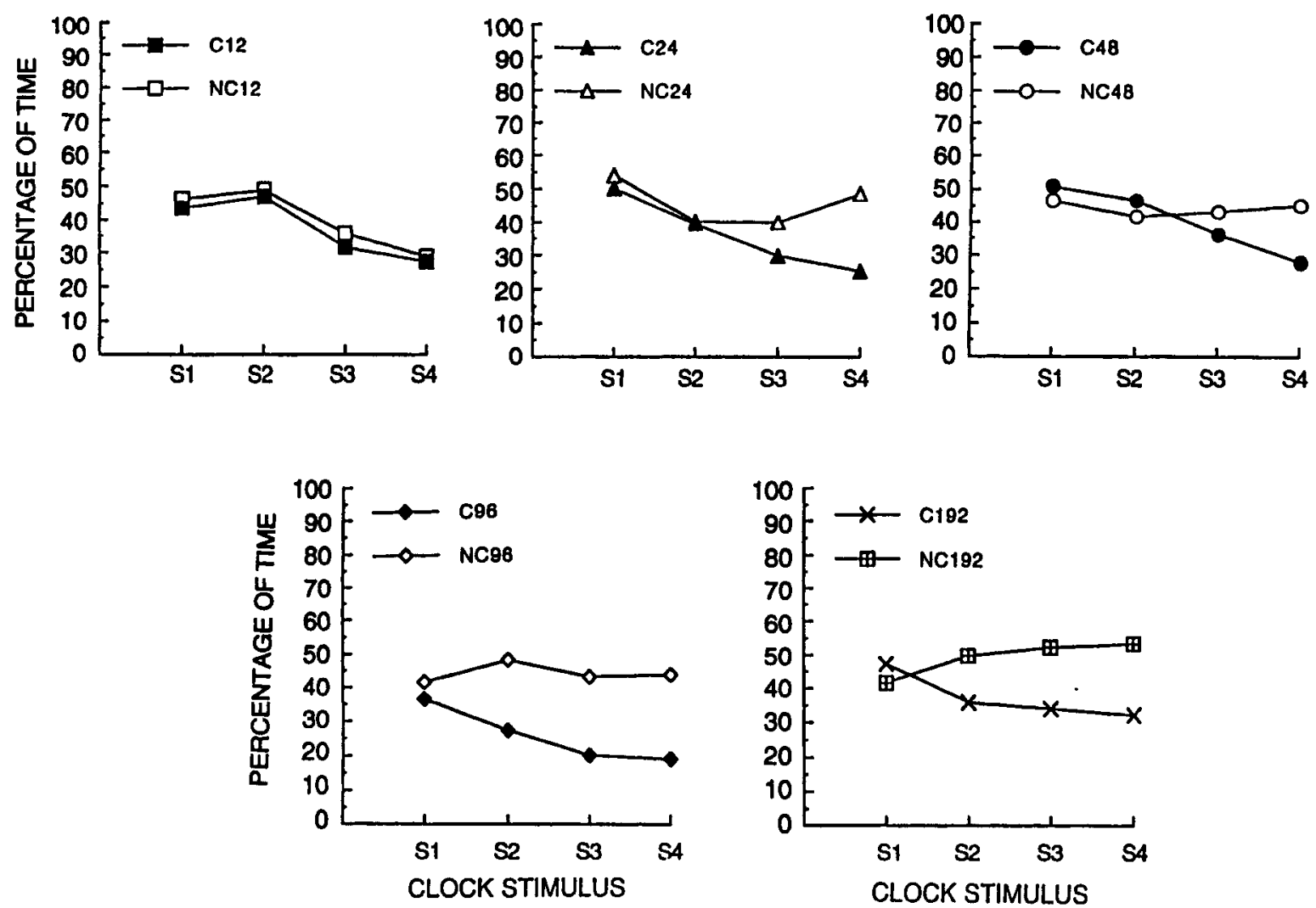

Figure 4. Mean percentage of time spent activating the near platform for each clock group and its corresponding no-clock group, averaged across the last 4 days of interfood clock training.

sures. Figures 5 and 6 show that the patterns and levels of activity on the far platform and of rearing were similar during the clock and no-clock conditions for all IFI lengths. Three-way ANOVAs examined the influence of IFI length $(12,24,48,96$, and $192 \mathrm{sec}$ ), group type (clock, no-clock), and segment of time in the IFI (i.e., clock stimulus for clock groups or comparable time period for the no-clock groups) on the levels of activity on the far platform and on rearing. The results revealed a main effect of segment of the IFI for activity on the far platform $[F \mathrm{~s}(3,210)>23.99]$ and only a two-way interaction between IFI length and segment of the IFI for rearing $[F \mathbf{s}(12,210)>2.47]$.

In terms of activity on the far platform (shown in Figure 5), the clock and no-clock groups showed similar patterns of responding across the IFI: Activity increased from the first to the second segment, followed by a decrease by the fourth segment during all IFI lengths. Although the initial three-way ANOVA failed to reveal a three-way interaction, individual ANOVAs were still conducted on the data from each clock group and its noclock counterpart in order to explore any significant differences not revealed in the previous analyses. Consistent with the previous analysis, the individual ANOVAs revealed only a main effect of segment in the IFI during each IFI length $[F \mathbf{s}(3,42)>9.76]$. Subsequent Scheffé tests revealed that each clock group and its no-clock counterpart showed an increase in activity from the first to the second segment, which then decreased by the last segment for all IFI lengths. That the individual ANOVAs failed to reveal any main effects of group type or any interactions between group type and time in the IFI during any IFI lengths shows that there were no differences in activity in the far platform between each clock group and its no-clock counterpart during any segment of the IFI.

Figure 6 shows that rearing generally decreased as the IFI elapsed for both clock and no-clock groups exposed to the 24- and 48-sec IFI lengths but changed very little during the other IFI lengths. Since the three-way ANOVA revealed a two-way interaction between IFI length and segment of the IFI for this response $[F(12,210)=2.47]$, individual ANOVAs were conducted on the data from each clock group and its no-clock counterpart in order to determine how responding changed across the IFI. Rearing peaked during $\mathrm{S} 1$ and decreased by $\mathrm{S} 4$ for rats exposed to the 24- and 48-sec IFIs, but rats exposed to the other IFI lengths failed to show significant changes across 


\section{FAR PLATFORM}
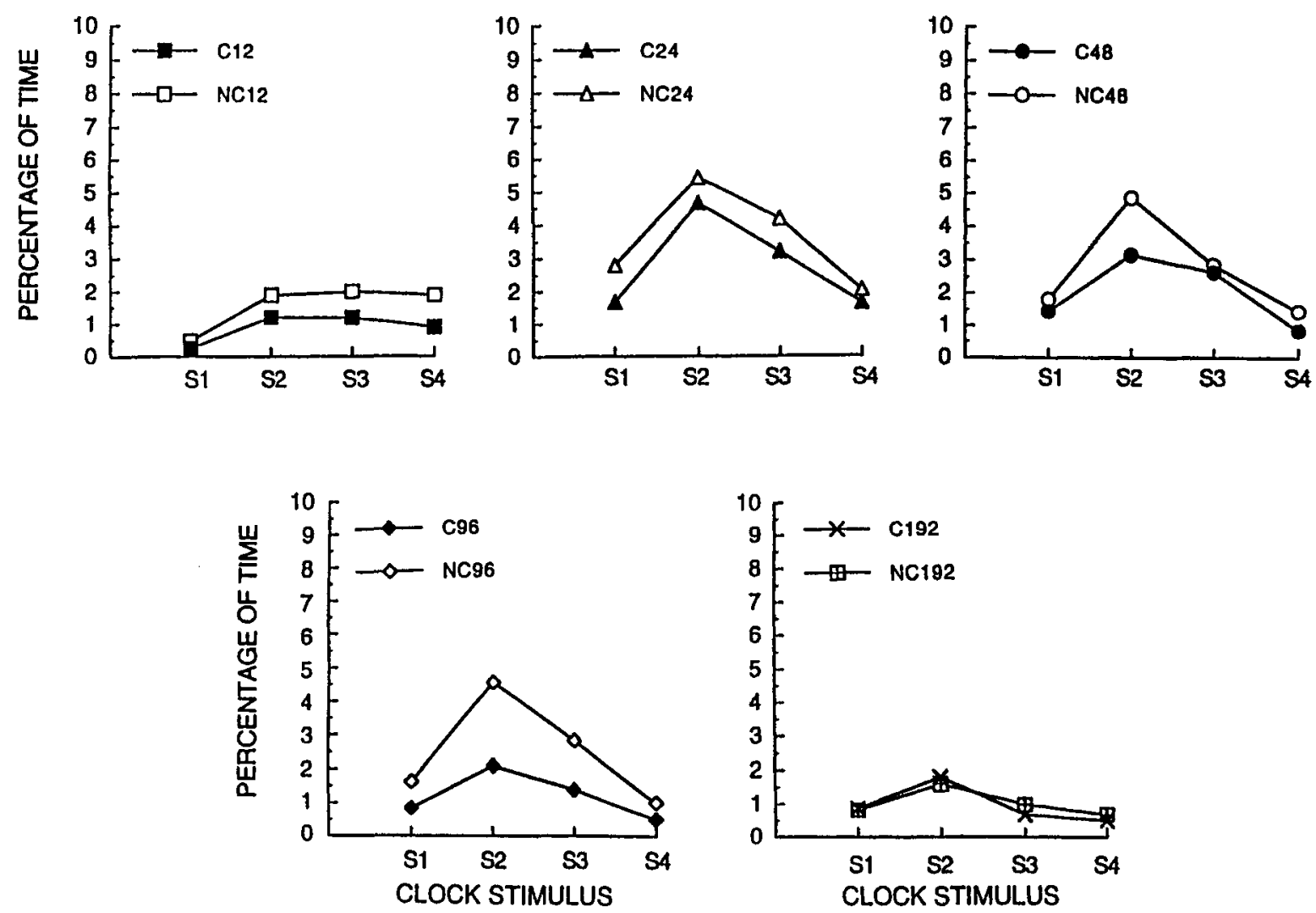

Figure 5. Mean percentage of time spent activating the far platform for each clock group and its corresponding no-clock group, averaged across the last 4 days of interfood clock training.

the IFI. Although it appears that rats exposed to the 12-sec IFI decreased rearing across the IFI, this effect only occurred in 3 of the 16 subjects. As was found for the measure of activity on the far platform, the individual ANOVAs failed to reveal any main effects of group type or any interactions between group type and time in the IFI during any IFI length.

\section{Scalar Versus Nonscalar Responding Across Clock Stimuli}

A second purpose of the present experiment was to determine which responses during the interfood clock were scalar and which were time bound. Figure 7 shows the time in the IFI at which each response peaked, graphed according to IFI length for each clock group. If a response is time bound (that is, peaks at the same average time after food delivery), the timing of response peaks should be similar for all IFI lengths. For example, a timebound response that peaks $15 \mathrm{sec}$ after food presentations should peak near that time during all IFI lengths. However, if a response is scalar, the time period at which it peaks should increase in proportion to the increase in IFI length. For example, a scalat response that peaks
$10 \mathrm{sec}$ after food during the 12 -sec IFI should peak $20 \mathrm{sec}$ after food during the 24-sec IFI and $40 \mathrm{sec}$ after food for the 48-sec IFI. The timing of the response peak was used to classify a response as scalar or time bound, because it was a criterion that could be used across all dependent measures (see Lucas et al., 1988, for a similar criterion). Other criteria, such as the onset of responding, could have been used for a terminal response, such as nosing in the feeder (Palya \& Pevey, 1987), that typically shows a gradual increase just before food. However, it would have been difficult to apply this criterion to responses, such as pawgrooming and rearing, that peak immediately after food and do not show the gradual increase across the IFI.

The top graphs of Figure 7 show that the time during which nosing in the feeder and activity on the far platform reached their maximum levels of responding (i.e., reached their peaks) increased in proportion with the increase in IFI length. Nosing in the feeder peaked approximately $10,20,41,84$, and $145 \mathrm{sec}$ after food presentations for Groups C12, C24, C48, C96, and C192, respectively. These time periods correspond to the time just after the onset of the last stimulus. The right top 


\section{REARING}
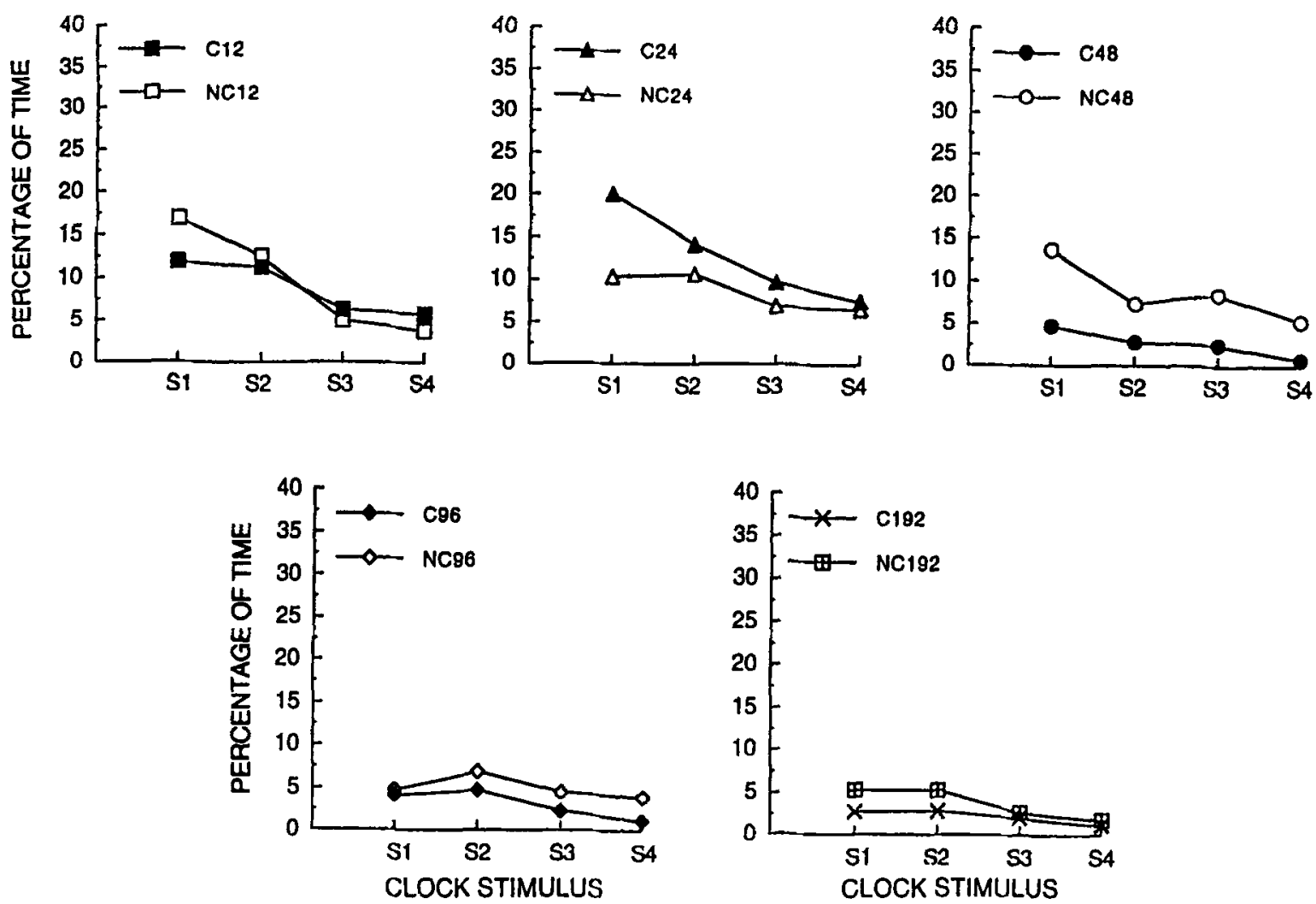

Figure 6. Mean percentage of time spent rearing for each clock group and its corresponding no-clock group during the last day of interfood clock training.

graph shows that activity on the far platform peaked about $4,8,15,28$, and $53 \mathrm{sec}$ after food presentations for Groups $\mathrm{C} 12, \mathrm{C} 24, \mathrm{C} 48, \mathrm{C} 96$, and $\mathrm{C} 192$, respectively. These times correspond to times approximately just after the onset of the second stimulus. In sum, the data suggest that nosing in the feeder and activity on the far platform are scalar, since the time at which they peaked increased in proportion to the increase in IFI length.

In contrast to nosing in the feeder and activity on the far platform, the remaining response measures were time bound. That is, each response peaked at about the same time after food presentation during different IFI lengths. The relatively flat line depicted in the bottom left graph of Figure 7 shows that activity on the near platform peaked about $5 \mathrm{sec}$ after food presentations during all IFI lengths, except for Group C96. Although it appears that this group peaked near $10 \mathrm{sec}$ after food presentation, examination of individual subjects revealed that the group average was inflated by 1 subject that peaked later than the other subjects. Because changes in pawgrooming across the IFIs were limited to the 96- and 192-sec IFI lengths and that changes in rearing were limited to the 24and 48 -sec IFI lengths, only data from these IFI lengths are shown. The middle bottom graph shows that rearing peaked about $6 \mathrm{sec}$ after food presentations, and the bottom right graph shows that pawgrooming peaked approximately $15 \mathrm{sec}$ after food presentations. Thus, rearing was time bound at shorter IFIs, whereas pawgrooming was time bound at longer IFIs.

\section{DISCUSSION}

The present results, produced by presenting periodic access to food during IFIs with and without an interfood clock, support previous findings that rats display a temporal organization of behavior across the IFI (Lucas et al., 1988; Reid et al., 1993). We measured several responses performed by the rats, and each response peaked at different average times within the IFI. Behavior near the feeder (i.e., standing on a platform in front of the feeder, rearing, and pawgrooming) peaked after food presentations; locomotion around the chamber (walking on the platform that was remote from the feeder) peaked in the the middle of the IFI; and nosing in the feeder peaked just before food presentations. On average, this order of responses was not affected by shortening or lengthening the IFI. 

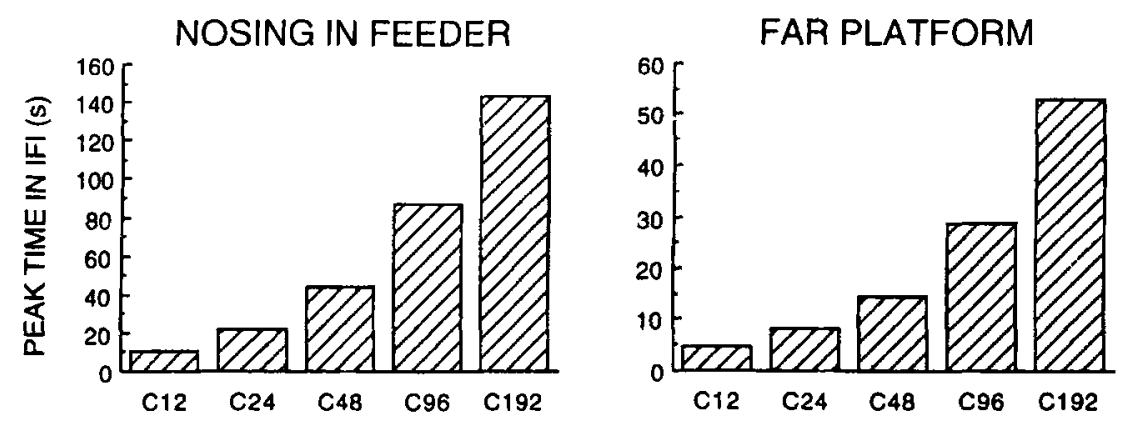

NEAR PLATFORM

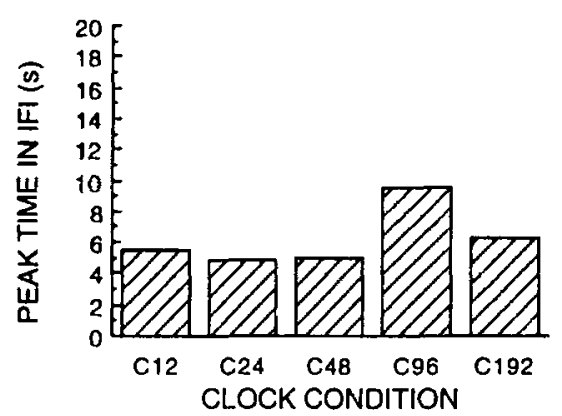

REARING

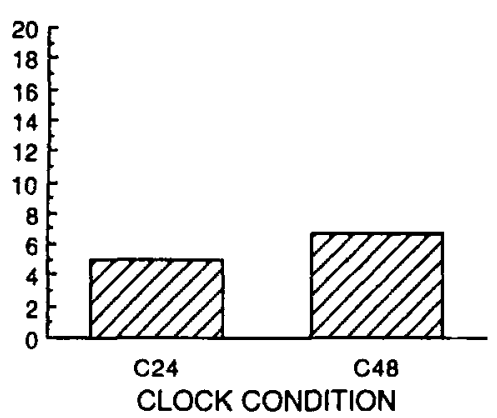

PAW GROOMING

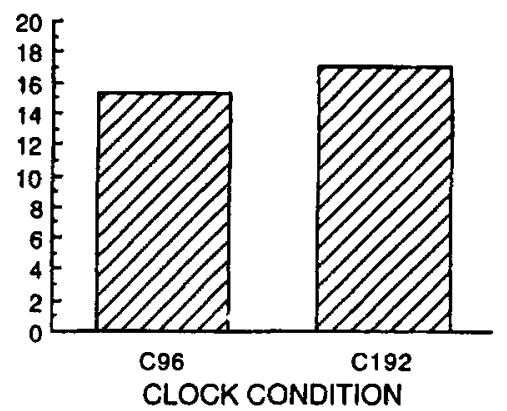

Figure 7. The time period in the interfood interval (IFI) during which each clock group reached its maximum level (i.e., reached its peak) of nosing in the the feeder, activity on the far platform, activity on the near platform, rearing, and pawgrooming, graphed according to IFI length.

One purpose of the present experiment was to determine how the presence of the interfood clock affected the temporal organization and amount of responding, as compared with the absence of the clock. Although the presence of the clock had little effect on the pattern and amount of locomotion and rearing, its presence facilitated nosing in the feeder and pawgrooming. This response-facilitating effect, however, was limited to particular quarters of the IFI and to particular IFI lengths. During intermediate IFIs of 24 and $48 \mathrm{sec}$, the last two clock stimuli facilitated nosing in the feeder above levels shown by groups that did not receive the clock. During the longest IFI of $192 \mathrm{sec}$, the first clock stimulus facilitated pawgrooming.

Another purpose of the present experiment was to determine which responses during the interfood clock showed scalar properties and which were time bound. Responses predominantly occurring in the middle and last half of the IFI showed scalar characteristics. Nosing in the feeder peaked during the last quarter of the IFI, regardless of IFI length, and activity on the far platform peaked in the middle of the second segment of the IFI, which suggests that these responses were scalar. However, postfood behavior, such as rearing and pawgrooming, displayed time-bound characteristics, reaching a maximum level about 6 and $15 \mathrm{sec}$ after food, respectively.

\section{Theoretical Accounts}

Because the present results showed that the effect of a clock stimulus depended on the IFI length, its position in the IFI, and the particular response being measured, a complete account of the present results must consider all of these factors. As we will discuss further, the effects cannot be fully understood with simple combinations of excitatory and inhibitory processes or by the use of response-classification frameworks alone. A more complete account of the results will require a combination of mechanisms and processes with ecologically based knowledge of behavior.

The sequence and location of responses across the interfood clock in this experiment were consistent with the behavior systems view that a sequence of different search modes and related responses occurs between periodic presentations of food (Timberlake, 1994; Timberlake \& Lucas, 1989). Recall that the behavior systems view argues that different responses should show peak expression at different times in the IFI. After ingestion of food, the animal is assumed to enter a postfood search mode that is characterized by an area-restricted search focused on the food site. Toward the middle of the IFI, the animal is assumed to enter a general search mode that is characterized by locomotion, taking the animal to a wider search area. Then, just before food presentation, the animal enters a focal search mode that is characterized by behavior focused on the food site. In the present experiment, the evidence for a general search mode consisted of a peak expression of locomotion away from the feeder during S2. Focal search behavior, in the form of nosing in the feeder, peaked just prior to the delivery of food. Postfood search consisted of standing near the feeder, with the addition of rearing and, perhaps, pawgrooming. 
The behavior systems view also suggests that responses and modes are potentially controlled by stimuli that are present during their expression. Thus, stimuli presented shortly before food should condition a focal search state and responses related to the procurement of imminent food (e.g., nosing in the feeder), whereas stimuli after food should condition a postfood focal search state related to searching for additional food, including small pieces that remain after eating (e.g., pawgrooming). The fact that the clock stimuli just before food (S3 and S4) facilitated nosing in the feeder and the clock stimulus after food (S1) facilitated postfood pawgrooming relative to conditions without the clock stimuli is consistent with a behavior systems view. Matthews et al. (1990) reported similar results with pigeons.

It is worth noting that the sequence of responses that emerged in the present experiment was also descriptively consistent with other frameworks that posit a sequence of response classes between presentations of food. For example, Staddon (1977; see also Staddon \& Ayres, 1975; Staddon \& Simmelhag, 1971) distinguished between interim responses that occur immediately after the receipt of the incentive (food), terminal responses, like pecking in pigeons or feeder-directed behavior in rats, that immediately precede the delivery of food, and facultative responses that occur between the interim and terminal responses. Similarly, Cohen et al. (1985) and Lawler and Cohen (1992) argued that nonterminal behavior could be divided into two classes. Time-locked behavior, represented in rats by pawgrooming, is hypothesized to have a repetitive, reflexive quality and to occur a fixed time after feeding. In contrast, general activity appears as gross motor activities and is predicted to expand in a constant proportion to the size of the interval.

One question that remains unanswered by these frameworks, however, is whether these responses are controlled by the explicit clock stimuli in the IFI. Probably because these frameworks were developed to account for responding during procedures in which animals receive food on periodic schedules without explicit predictive stimuli, they make no specific predictions applicable to the interfood clock.

Although the behavior systems approach (Timberlake \& Lucas, 1989) makes specific predictions about which responses should be controlled by which clock stimuli, the control actually exerted by the clock stimuli was more complex than it was in this initial view. First, the ability of a clock stimulus to facilitate nosing in the feeder and pawgrooming depended on the length of the IFI. The clock stimuli just before food presentations facilitated nosing in the feeder during the intermediate IFI lengths only, and the clock stimulus just after food presentations facilitated pawgrooming during the longest IFI length only.

To provide a complete explanation of the constellation of results obtained, it may be worthwhile to acquire a more explicit understanding of the rats' ecology and of how underlying foraging mechanisms map to the current procedures and apparatus (Timberlake, 1983; Timberlake \& F. J. Silva, 1994). An ecologically based approach assumes that the behavior of a hungry rat in an experimental chamber is evolutionarily based on evolved mechanisms that can facilitate locating and consuming food, even in an artificial environment, if it is appropriately tuned (Timberlake, 1983, 1986, 1994; Zeiler, 1991). For example, Zeiler suggested that the ability of an external cue to control behavior may depend on food density. From this perspective, there is less reason for a foraging animal to attend closely to external cues that predict food when food is abundant or competition is minimal. However, if food is less abundant, animals might benefit by attending closely to predictive cues. When the density of food falls below some threshold, an alternate foraging strategy might be evoked, such as leaving the current patch for another (see, also, Krebs \& Inman, 1992).

In the case of nosing in the feeder of the clock and noclock groups, an ecological account suggests that rats may not readily attend as carefully to predictive cues when food is abundant (e.g., 12-sec IFIs), but should attend to predictive cues when food is less abundant (e.g., 24- and 48-sec IFIs). The failure of the clock stimulus to produce more responding in the 96- and 192-sec IFIs may be a motivational issue related to density of reward or a question of attention to external cues. In the presence of predictable IFI durations, rats may not as readily associate external cues with food at low overall food densities (though this is not so in the case of unpredictable IFI durations; see Gibbon, Farrell, Locurto, Duncan, \& Terrace, 1980; Gonzalez, 1974). Future experiments could assess these hypotheses more directly by increasing the food density after longer IFIs (96 and $192 \mathrm{sec}$ ), so that it. is similar to the density during shorter IFIs, and/or decreasing the predictability of the IFI.

It is less clear how the facilitation of a nonconsummatory response, such as pawgrooming at the longest IFI, serves to increase the probability of finding food, but it should be remembered that an important issue is the fit between the mechanisms that serve to regulate behavior and the experimental procedure. One possible explanation can be found in Lucas et al.'s (1988) suggestion that drinking, rearing, and grooming responses are transitional behaviors between the two dominant types of behavior-postfood area-restricted search and general search. At shorter IFIs, there is insufficient time for the expression of area-restricted search, general search, and transitional behaviors. However, as the interval grows longer, the opportunity for expression of the transitional behaviors increases. A related possibility is that the absolute motivation of such transitional behavior may increase, because, with an IFI over $3 \mathrm{~min}$, a postfood stimulus may signal the end of a feeding bout, thus evoking parts of postmeal behavior, such as drinking and grooming. At very short IFIs, the addition of a clock stimulus after food may simply signal imminent food, engaging area-restricted search rather than postfood-related behavior. During longer IFIs, the cue may become associ- 
ated with processes related to the (temporary) termination of a feeding bout, facilitating grooming and rearing. Thus, the first clock stimulus may control different combinations of states during very short, intermediate, and long IFIs.

Lastly, a complete account of the present results should explain why the presence of the clock stimuli did not increase remote locomotion and rearing. On the basis of Timberlake and Lucas's (1989) behavior systems account, we expected that stimuli presented temporally distant from food (e.g., S2 or S3) would control and, presumably, facilitate a general search mode, expressed through remote locomotion. However, the present results showed that the clock stimuli did not significantly increase locomotion (i.e., activity on the far platform). Recent results from K. M. Silva and Timberlake (1997) have suggested that general search responses may not be under tight control by an external stimulus. Matthews and Lerer (1987) also showed that the general pacing behavior of pigeons in the middle third of the IFI was not well controlled by external stimuli. Similarly, Domjan (1994) provided data indicating that general search may be more controlled by general, contextual cues than by discrete stimuli. The persistence of a general search mode makes sense from an ecological standpoint in that, once aroused, a general search mode must sustain an animal's behavior over some period without reward or strongly correlated external stimuli. Otherwise, a hungry animal might extinguish search behavior in the absence of immediate food (K. M. Silva \& Timberlake, 1997). However, a test of this possibility will require a specific probe for the existence of a general motivational state related to the second quarter of the IFI (K. M. Silva \& Timberlake, in press).

\section{Traditional Associative Accounts}

A traditional associative view of Pavlovian conditioning posits that, when the temporal distance between the CS and US is increased beyond a short, optimal length, the probability of the conditioned response decreases (Schneiderman \& Gormezano, 1964). This decline in responding is typically interpreted as a diminution in control or as an absence of control by temporally distant CSs. That nosing in the feeder was relatively low at the beginning of the IFI and then increased over the last two clock stimuli preceding food, as compared with groups without the clock, is consistent with the view that learned responding depends on the degree to which the signal is contiguous with food presentations (Gibbon \& Balsam, 1981; Rescorla \& Wagner, 1972). However, the behavior systems view argues that the decline in responding is not due to an absence of control by temporally distant CSs but to their control of a repertoire of responses that differ from those typically measured. Consistent with this latter view are the results showing that a temporally distant clock stimulus ( $\mathrm{S} 1$ ) evoked more pawgrooming than did more temporally contiguous clock stimuli (S2, S3, and S4) and more than that for a group that did not receive the clock.
Several other aspects of the results are related to a more traditional association-based view of behavior. For example, the decrease in nosing in the feeder and the increase in movement away from the feeder after food presentations raise the possibility that associative inhibition may have contributed to the results of the present experiments (Papadouka \& Matthews, 1995; Wasserman, Franklin, \& Hearst, 1974). However, if the clock stimuli after food accrued inhibitory properties, the clock groups should have nosed less in the feeder than did the no-clock groups, a result that did not occur.

That nosing in the feeder peaked at the onset of the last clock stimulus during the intermediate IFI lengths is consistent with Gibbon and Balsam's (1981) suggestion that stimuli during the last half of the IFI provide positive information about the delivery of food and that the amount of responding during these stimuli is determined by the relative time in the interval. However, strictly speaking, their account applies only to procedures that partition the trial into two components-one during which no food is presented and the other contiguous with food presentation - and assumes that only the final contiguous stimulus controls responding. This implication was borne out neither by the present data nor by other studies (Palya, 1985). At the intermediate IFI lengths, the clock stimuli during the last half of the IFI controlled nosing in the feeder to some extent. Furthermore, at the longest IFI, the first clock stimulus controlled a qualitatively different response.

An explanation for the ability of stimuli before and after food presentations to control responses, although stimuli in the middle of the IFI do not, may be found within the framework of Palya's (1993) bipolar model of interfood clock performance. This approach specifies that stimuli correlated with the successive portions of the latter half of the IFI are increasingly positive, stimuli correlated with successively earlier portions of the IFI are increasingly negative, the stimuli in the middle of the IFI are relatively neutral, and qualitative changes in behavior are expected to stimuli correlated with these various portions of the IFI. That stimuli during the last half of the IFI controlled one response (nosing in the feeder) and stimuli at the beginning of the IFI controlled a different response (pawgrooming) is consistent with the bipolar account. However, like previous models that predict the form of responding across an IFI (Timberlake \& K. M. Silva, 1995), the bipolar model did not predict that the control would depend on the IFI length. That the clock stimuli in the middle of the IFI did not control the response with which it was correlated (locomotion) is uniquely accounted for by the bipolar approach, since it predicts that the control exerted by clock stimuli should be weakest at this point. It is clear that the patterning of responding poses a complex question for all accounts.

\section{Conclusions}

In summary, the constellation of effects observed in the present study shows that rat behavior possesses a par- 
ticular sequential and temporal organization during periodic presentations of food. On average, behavior near the feeder peaks after food presentations in a time-bound fashion, whereas locomotion around the chamber peaks near the middle of the IFI and nosing in the feeder peaks just before food, both in a scalar fashion. The present study also provides information about how the presence of external timing cues affects the expression of behavior. In different respects, the data are compatible with views of scalar timing (Gibbon, 1977; Gibbon \& Balsam, 1981 ) and with the response-classification frameworks of Cohen et al. (1985), of Staddon (1977), and of Timberlake and Lucas (1989). However, only a version of Timberlake and Lucas (1989) and Lucas et al. (1988) offered an even marginally inclusive account of how the interfood clock and IFI length interacted in affecting the expression of multiple responses. A more complete account of behavior during periodic food presentations must consider that responses cluster in sequential groups, that different responses possess different timing characteristics, and that some responses are better controlled by relatively punctate external stimuli than are other responses. For those responses controlled by external stimuli, the control may depend on both absolute and relative temporal intervals between the stimulus and food and between one food presentation and the next. The present paper suggests that these effects might be better understood by continued development of ecologically based accounts of learning and behavior that combine the organization and processes of behavior systems, the specific feeding ecology of the rat, and the experimental circumstances and paradigms of traditional learning.

\section{REFERENCES}

BARNET, S. A. (1975). The rat: A study in behavior. Chicago: Chicago University Press.

Campagnoni, F. R., Lawler, C. P., \& Cohen, P. S. (1986). Temporal patterns of reinforcer-induced general activity and attack in pigeons. Physiology \& Behavior, 37, 577-582.

Cohen, P. S., Looney, T. A., Campagnoni, F. R., \& Lawler, C. P. (1985). A two-state model of reinforcer-induced motivation. In F. R. Brush \& J. B. Overmier (Eds.), Affect, conditioning, and cognition: Essays on the determinants of behavior (pp. 281-297). Hillsdale, NJ: Erlbaum.

DinsmoOR, J. A. (1994). A comparison between the block clock and standard autoshaping procedures. Learning \& Motivation, 25, 313337

Dinsmoor, J. A., Dougan, J. D., Pfister, J., \& Thiels, E. (1992). The autoshaping procedure as a residual block clock. Journal of the Experimental Analysis of Behavior, 58, 265-276.

Dinsmoor, J. A., LEE, D. M., \& Brown, M. M. (1986). Escape from serial stimuli leading to food. Journal of the Experimental Analysis of. Behavior, 46, 259-279.

Domjan, M. (1994). Formulation of a behavior system for sexual conditioning. Psychonomic Bulletin \& Review, 1, 421-428.

FERSTER, C. B., \& SKINNER, B. F. (1957). Schedules of reinforcement. New York: Appleton-Century-Crofts.

GibBON, J. (1977). Scalar expectancy theory and Weber's law in animal timing. Psychological Review, 84, 279-235.

GibBon, J., \& Balsam, P. (1981). Spreading association in time. In C. M. Locurto, H. S. Terrace, \& J. Gibbon (Eds.), Autoshaping and conditioning theory (pp. 218-253). New York: Academic Press.
Gibbon, J., Farrell, L., Locurto, C. M., Duncan, H. J., \& Terrace, H. S. (1980). Partial reinforcement in autoshaping with pigeons. Animal Learning \& Behavior, 8, 45-59.

GonZaLEZ, F. A. (1974). Effects of varying the percentage of key illumination paired with food in a positive automaintenance procedure. Journal of the Experimental Analysis of Behavior, 22, 483-489.

KaZDIN, A. E. (1982). Single-case research designs: Methods for clinical and applied settings. New York: Oxford University Press.

KilleEN, P. R. (1975). On the temporal organization of behavior. Psychological Review, 82, 80-115.

KREBS, J. R., \& INMAN, A. J. (1992). Learning and foraging: Individuals, groups, and populations. In L. Real (Ed.), Behavioral mechanisms in evolutionary ecology (pp. 47-65). Chicago: University of Chicago Press.

LAWLer, C. P., \& Cohen, P. S. (1992). Temporal patterns of scheduleinduced drinking and pawgrooming in rats exposed to periodic food. Animal Learning \& Behavior, 20, 266-280.

Lucas, G. A., Timberlake, W., \& Gawley, D. J. (1988). Adjunctive behavior of the rat under periodic food delivery in a 24-hour environment. Animal Learning \& Behavior, 16, 19-30.

MaTThews, T. J., BoRDI, F., \& DePOLlo, D. (1990). Schedule-induced kinesic and taxic behavioral stereotypy in the pigeon. Journal of Experimental Psychology: Animal Behavior Processes, 16, 335-344.

MatThews, T. J., \& LERER, B. E. (1987). Behavior patterns in pigeons during autoshaping with an incremental conditioned stimulus. Animal Learning \& Behavior, 15, 69-75.

PaLYa, W. L. (1985). Sign-tracking with an interfood clock. Journal of the Experimental Analysis of Behavior, 43, 321-330.

PaLYA, W. L. (1993). Bipolar control in fixed interfood intervals. Journal of the Experimental Analysis of Behavior, 60, 354-359.

PALYA, W. L., \& BEVINS, R. A. (1990). Serial conditioning as a function of stimulus, response, and temporal dependencies. Journal of the $E x$ perimental Analysis of Behavior, 53, 65-85.

Palya, W. L., \& Pevey, M. E. (1987). Serial conditioning as a function of parametric variations of an interfood clock. Animal Learning \& Behavior, 15, 249-262.

Papadouka, V., \& Matthews, T. J. (1995). Motivational mechanisms and schedule-induced behavioral stereotypy. Animal Learning \& Behavior, 23, 461-469.

ReID, A. K., BaCHA, G., \& Moran, C. (1993). The temporal organization of behavior on periodic food schedules. Journal of the Experimental Analysis of Behavior, 59, 1-27.

REID, A. K., \& STADDON, J. E. R. (1990). Mechanisms of schedule entrainment. In S. J. Cooper \& C. T. Dourish (Eds.), Neurobiology of stereotyped behavior (pp. 200-231). London: Oxford University Press.

Rescorla, R. A., \& WAGNER, A. R. (1972). A theory of Pavlovian conditioning: Variations in effectiveness of reinforcement and nonreinforcement. In A. H. Black \& W. F. Prokasy (Eds.), Classical conditioning II: Current research and theory (pp. 64-99). New York: Appleton-Century-Crofts.

Schneiderman, N., \& Gormezano, I. (1964). Conditioning of the nictitating membrane of the rabbit as a function of the CS-US interval Journal of Comparative \& Physiological Psychology, 69, 188-195.

Silva, F. J., Timberlake, W., \& Koehler, T. L. (1996). A behavior systems approach to bidirectional excitatory serial conditioning. Learning \& Motivation, 27, 150-160.

Silva, K. M. (1996). Appetitive behavior during an interfood clock. Unpublished doctoral dissertation, Indiana University, Bloomington.

Silva, K. M., \& Timberlake, W. (1997). A behavior systems view of conditioned states during long and short CS-US intervals. Learning \& Motivation, 28, 465-490.

Silva, K. M., \& Timberlake, W. (in press). A behavior systems view of responding to probe stimuli during an interfood clock. Animal Learning \& Behavior

Staddon, J. E. R. (1977). Schedule-induced behavior. In W. K. Honig \& J. E. R. Staddon (Eds.), Handbook of operant behavior (pp. 125152). New York: Prentice-Hall.

Staddon, J. E. R., \& Ayres, S. L. (1975). Sequential and temporal properties of behavior induced by a schedule of periodic food delivery. Behaviour, 54, 26-49. 
Staddon, J. E. R., \& Simmelhag, V. L. (1971). The "superstition" experiment: A reexamination of its implications for the principles of adaptive behavior. Psychological Review, 78, 3-43.

TimberlaKe, W. (1983). The functional organization of appetitive behavior: Behavior systems and learning. In M. D. Zeiler \& P. Harzem (Eds.), Advances in the analysis of behavior: Vol. 3. Biological factors in learning (pp. 177-221). Chichester, U.K.: Wiley.

TimberLaKe, W. (1986). Unpredicted food produces a mode of behavior that affects rats' subsequent reactions to a conditioned stimulus: A behavior-system approach to "context blocking." Animal Learning \& Behavior, 14, 276-286.

TimBerlaKe, W. (1994). Behavior systems, associationism, and Pavlovian conditioning. Psychonomic Bulletin \& Review, 1, 405-420.

TimberLake, W., \& LuCAS, G. A. (1985). The basis of superstitious behavior: Chance contingency, stimulus substitution, or appetitive behavior? Journal of the Experimental Analysis of Behavior, 44, 279-299.

Timberlake, W., \& LuCAS, G. A. (1989). Behavior systems and learning: From misbehavior to general principles. In S. B. Klein \& R. R. Mower (Eds.), Contemporary learning theories: Instrumental conditioning theory and the impact on biological constraints on learning (pp. 237-275). Hillsdale, NJ: Erlbaum.
TymberlaKe, W., \& LuCAS, G. A. (1991). Periodic water, interwater interval, and adjunctive behavior in a 24 -hour multiresponse environment. Animal Learning \& Behavior, 19, 369-380.

Timberlake, W., \& Silva, F. J. (1994). Observation of behavior, inference of function, and the study of learning. Psychonomic Bulletin \& Review, 1, 73-88.

Timberlake, W., \& Silva, K. M. (1995). Appetitive behavior in psychology, ethology, and behavior systems. In N. Thompson (Ed.), Perspectives in ethology: Vol. 11. Behavioral design (pp. 212-254). New York: Plenum.

Wasserman, E. A., Franklin, S. R., \& Hearst, E. (1974). Pavlovian appetitive contingencies and approach versus withdrawal to conditioned stimuli in pigeons. Journal of Comparative \& Physiological Psychology, 86, 616-627.

ZEILER, M. D. (1991). Ecological influences on timing. Journal of Experimental Psychology: Animal Behavior Processes, 17, 13-25.

(Manuscript received April 9, 1997, revision accepted for publication December 18,1997 .) 